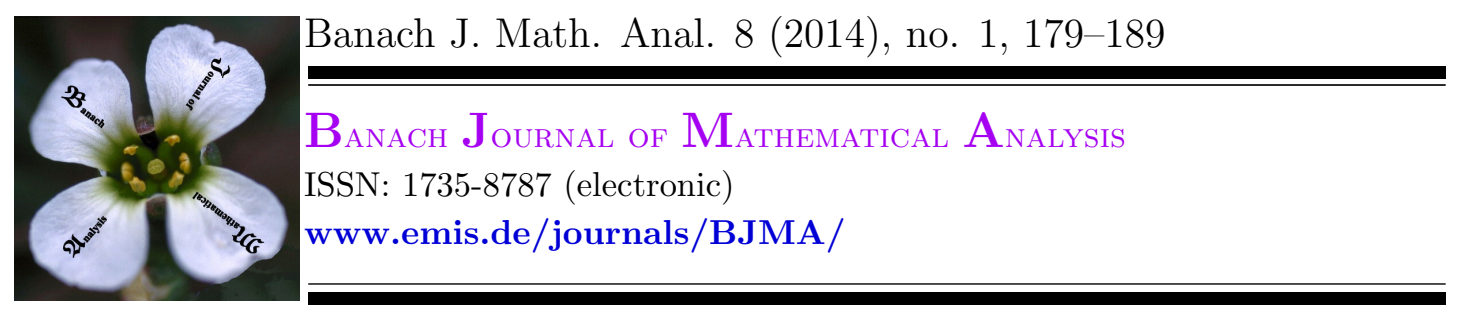

\title{
THE PROBLEM OF ISOMETRIC EXTENSION IN THE UNIT SPHERE OF THE SPACE $s_{p}(\alpha, H)$
}

\author{
XIAOHONG FU \\ Communicated by D. E. Alspach
}

\begin{abstract}
In this paper, we consider the problem of isometric extension in the unit sphere of the space $s_{p}(\alpha, H)$. We obtain that Lamperti isometric mapping of the unit sphere $S\left(s_{p}(\alpha, H)\right)$ into itself can be extended to an isometry on the whole space $s_{p}(\alpha, H)$.
\end{abstract}

\section{INTRODUCTION}

Let $E$ and $F$ be normed spaces. A mapping $V: E \rightarrow F$ is called an isometry if $\|V x-V y\|=\|x-y\|$ for all $x_{1}, x_{2} \in E$ (see, e.g., [10]). The classical MazurUlam theorem in [11] describes the relation between isometry and linearity and states that every onto isometry $V$ between two normed spaces with $V(0)=0$ is linear. So far, this has been generalized in several directions (see, e.g., [14]). One of them is the study of the isometric extension problem.

Mankiewicz in [9] showed that an isometry which maps a connected subset of a normed space $X$ onto an open subset of another normed space $Y$ can be extended to an affine isometry from $X$ to $Y$. In 1987, Tingley [17] posed the problem of extending an isometry between unit spheres as follows.

Let $E$ and $F$ be two real Banach spaces. Suppose that $V_{0}$ is a surjective isometry between the two unit spheres $S_{1}(E)$ and $S_{1}(F)$. Is $V_{0}$ necessarily a restriction of a linear or affine transformation to $S_{1}(E)$ ?

Date: Received: 19 March 2013 ; Accepted: 1 June 2013.

2010 Mathematics Subject Classification. Primary 46B04; Secondary 46B20, 46E40.

Key words and phrases. Sobolev space, isometric mapping, isometric extension. 
It is very difficult to answer this question, even in two dimensional cases. In the same paper, Tingley proved that if $E$ and $F$ are finite-dimensional Banach spaces and $V_{0}: S_{1}(E) \rightarrow S_{1}(F)$ is a surjective isometry, then $V_{0}(x)=-V_{0}(-x)$ for all $x \in S_{1}(E)$. In [3], Ding gave an affirmative answer to Tingley problem, when $E$ and $F$ are Hilbert spaces. In the case when $E$ and $F$ are some metric vector spaces, the corresponding extension problem was investigated in $[1,5]$. See also $[6,7,8,12,15,17]$ for some related results.

By $s_{p}(\alpha), 1 \leq p<\infty, p \neq 2, \alpha \geq 0$, is denoted the discrete analogue of the classical Sobolev space, which is the linear space of all real or complex sequences $x=(x(k))_{k \in \mathbf{N}}$ for which

$$
\|x\|=\left(\sum_{k=1}^{\infty}|x(k)|^{p}+\alpha \sum_{k=1}^{\infty}|x(k+1)-x(k)|^{p}\right)^{\frac{1}{p}}<\infty .
$$

It is clear that $s_{p}(0)=l^{p}$. If $\alpha>0$, then since

$$
\|x\|_{l^{p}} \leq\|x\| \leq\left(1+2^{p} \alpha\right)^{1 / p}\|x\|_{l^{p}}
$$

where $\|x\|_{l^{p}}$ is the standard $l^{p}$ norm, it follows that $s_{p}(\alpha)$ is isomorphic to $l^{p}$, but these spaces are not isometric.

If an isometry on $s_{p}(\alpha)$ preserves both sums in formula (1.1), then it is called the Lamperti isometry (see [2]). From the definition we see that Lamperti isometry is independent of $\alpha$ for the space $s_{p}(\alpha)$.

Define $s_{p}(\alpha, H)(1 \leq p<\infty, p \neq 2, \alpha \geq 0)$ to be the collection of all element sequences in the Hilbert space $H$ such that

$$
\|x\|=\left(\sum_{k=1}^{\infty}\|x(k)\|^{p}+\alpha \sum_{k=1}^{\infty}\|x(k+1)-x(k)\|^{p}\right)^{\frac{1}{p}}<\infty .
$$

If an isometry on $s_{p}(\alpha, H)$ preserves both sums in formula (1.2), then it is called the Lamperti isometry. From the definition we see that Lamperti isometry is independent of $\alpha$ for the space $s_{p}(\alpha, H)$.

Define $l^{p}(H)(1 \leq p<\infty, p \neq 2)$ to be the collection of all element sequences in the Hilbert space $H$ such that

$$
\|x\|=\left(\sum_{k=1}^{\infty}\|x(k)\|^{p}\right)^{\frac{1}{p}}<\infty .
$$

In [4], Fleming and Jamison proved that for $1<p<\infty, \alpha>0, \alpha \neq 1$, the only surjective isometries on $s_{p}(\alpha)$ are scalar multiples of the identity. In [2], it is shown that there are no Lamperti isometries of finite codimension that are not surjective, and two distinct examples are given. In this paper, we study the problem of isometric extension in the unit sphere of the space $s_{p}(\alpha, H)$. We prove that if $V_{0}$ is an isometric mapping on the unit sphere of the subspace $E$ of $s_{p}(\alpha, H)$ :

$$
\operatorname{supp} E=\{k(n): k(n+1)-k(n)>1, \quad n \in \mathbf{N}\}
$$


and

$$
e_{x(n)}=\underbrace{(o, \cdots, x(n), o, \cdots)}_{\mathrm{n}-\text { th place }} \in E,
$$

then it can be extended to an isometry on the whole space $E$. Also, we prove that Lamperti isometric mapping on the unit sphere $S\left(s_{p}(\alpha, H)\right)$ into itself can be extended to an isometry on the whole space $s_{p}(\alpha, H)$.

\section{MAin RESUlTs}

In this section, we give our main results. For this purpose, we need some lemmas that will be used in the proofs of our main results. We begin with the following result.

Lemma 2.1. If $V_{0}$ is an isometric mapping from the unit sphere $S\left(s_{p}(\alpha, H)\right)$ into $S\left(s_{p}(\alpha, H)\right)$, then

$$
V_{0}(-x)=-V_{0}(x), \quad \text { for all } x \in S\left(s_{p}(\alpha, H)\right) .
$$

Proof. It is easy to see that the space $s_{p}(\alpha, H)$ is strictly convex, since $s_{p}(\alpha)$ and the Hilbert space $\mathrm{H}$ are strictly convex spaces(in fact, from [4], $s_{p}(\alpha)$ is uniformly convex and smooth). That is, if $\|u+v\|=\|u\|+\|v\|$, then $u=c v$ for some $c>0$. From this and since

$$
\left\|V_{0}(-x)-V_{0}(x)\right\|=\|-x-x\|=2=\left\|V_{0}(-x)\right\|+\left\|-V_{0}(x)\right\|,
$$

we have that $V_{0}(-x)=-c V_{0}(x)$ for some $c>0$. This implies $|c|=1$, from which the lemma follows.

Lemma 2.2. If $x, y \in l^{p}(H)(1 \leq p<\infty, p \neq 2)$, then

$$
\|x+y\|^{p}+\|x-y\|^{p}=2\left(\|x\|^{p}+\|y\|^{p}\right) \Leftrightarrow \operatorname{supp} x \cap \text { supp } y=\emptyset \text {, }
$$

where supp $x=\{n: x(n) \neq 0, n \in \mathbf{N}\}$.

Proof. The sufficiency is trivial. Following, we prove the necessity.

Suppose that $x=\{x(n)\}$ and $y=\{y(n)\}$ are elements in $l^{p}(H)$. Since $H$ is a Hilbert space, we have

$$
\|x(n)+y(n)\|^{2}+\|x(n)-y(n)\|^{2}=2\left(\|x(n)\|^{2}+\|y(n)\|^{2}\right) .
$$

We will divide the proof into two steps.

Firstly, we prove if $0<p<2$

$$
\|x(n)+y(n)\|^{p}+\|x(n)-y(n)\|^{p} \leq 2\left(\|x(n)\|^{p}+\|y(n)\|^{p}\right),
$$

while if $p>2$

$$
\|x(n)+y(n)\|^{p}+\|x(n)-y(n)\|^{p} \geq 2\left(\|x(n)\|^{p}+\|y(n)\|^{p}\right)
$$

and the equality holds if and only if $\|x(n)\|\|y(n)\|=0$. 
Indeed, if $0<p<2$, the function $\varphi(u)=u^{\frac{p}{2}}(u \geq 0)$ satisfies the following inequality

$$
\varphi(\alpha+\beta) \leq \varphi(\alpha)+\varphi(\beta)
$$

and the equality holds if and only if $\alpha \beta=0$.

Since $\varphi$ is concave on $[0, \infty)$, it follows from (2.1) and (2.4) that

$$
\begin{aligned}
& \frac{1}{2}\left(\|x(n)+y(n)\|^{p}+\|x(n)-y(n)\|^{p}\right) \\
= & \frac{1}{2}\left(\varphi\left(\|x(n)+y(n)\|^{2}\right)+\varphi\left(\|x(n)-y(n)\|^{2}\right)\right) \\
\leq & \varphi\left(\frac{\|x(n)+y(n)\|^{2}+\|x(n)-y(n)\|^{2}}{2}\right) \\
= & \varphi\left(\|x(n)\|^{2}+\|y(n)\|^{2}\right) \\
\leq & \varphi\left(\|x(n)\|^{2}\right)+\varphi\left(\|y(n)\|^{2}\right) \\
= & \|x(n)\|^{p}+\|y(n)\|^{p} .
\end{aligned}
$$

The equality holds if and only if $\|x(n) \mid\|\|y(n)\|=0$.

For the case $p>2, \varphi$ is convex on $[0, \infty)$. All the reverse inequalities hold in (2.4) and (2.5).

Then, we can prove the necessity of Lemma 2.2 by the definition of norm in the space $l^{p}(H)$ and the result from the first step.

Lemma 2.3. Let $V_{0}$ be an isometric mapping from the unit sphere $S\left(l^{p}(H)\right)$ into $S\left(l^{p}(H)\right)(1<p<\infty, p \neq 2)$. Then

$$
(\operatorname{supp} x) \cap(\operatorname{supp} y)=\emptyset \Leftrightarrow\left(\operatorname{supp} V_{0}(x)\right) \cap\left(\operatorname{supp} V_{0}(y)\right)=\emptyset .
$$

Proof. By the assumption of $V_{0}$, we have

$$
\left\|V_{0}(x)-V_{0}(y)\right\|=\|x-y\| .
$$

Since $l^{p}(H)$ is strictly convex, it follows from the proof of Lemma 2.1 that,

$$
V_{0}(-x)=-V_{0}(x)
$$

and thus

$$
\left\|V_{0}(x)+V_{0}(y)\right\|=\left\|V_{0}(x)-V_{0}(-y)\right\|=\|x+y\| .
$$

Notice that (2.6) and(2.7) assure that

$$
\|x+y\|^{p}+\|x-y\|^{p}=2\left(\|x\|^{p}+\|y\|^{p}\right)
$$

and

$$
\left\|V_{0}(x)+V_{0}(y)\right\|^{p}+\left\|V_{0}(x)-V_{0}(y)\right\|^{p}=2\left(\left\|V_{0}(x)\right\|^{p}+\left\|V_{0}(y)\right\|^{p}\right)
$$

hold simultaneously. According to Lemma 2.2, we obtain

$$
(\operatorname{supp} x) \cap(\operatorname{supp} y)=\emptyset \Leftrightarrow\left(\operatorname{supp} V_{0}(x)\right) \cap\left(\operatorname{supp} V_{0}(y)\right)=\emptyset .
$$

The following result is similar to that in [4]. 
Lemma 2.4. Let $V_{0}$ be an isometric mapping from the unit sphere $S\left(s_{p}(\alpha, H)\right)$ into $S\left(s_{p}(\alpha, H)\right)$ and $R$ be the right-translation operator, that is,

$$
R x=(0, x(1), x(2), \cdots, x(n), \cdots), \forall x=\{x(n)\} \in s_{p}(\alpha, H) .
$$

If

$$
\left\{\begin{array}{l}
\text { supp } x \cap \text { supp } y=\emptyset \\
\text { supp } x \cap \text { supp } R(y)=\emptyset \\
\text { supp } R(x) \cap \operatorname{supp} y=\emptyset
\end{array}\right.
$$

then

$$
\left\{\begin{array}{l}
\text { supp } V_{0}(x) \cap \operatorname{supp} V_{0}(y)=\emptyset \\
\text { supp } V_{0}(x) \cap \operatorname{supp} R\left(V_{0}(y)\right)=\emptyset \\
\text { supp } R\left(V_{0}(x)\right) \cap \operatorname{supp} V_{0}(y)=\emptyset
\end{array}\right.
$$

Remark 2.5. By the definition of the norm in the space $s_{p}(\alpha, H)$ and the condition of (2.8), it is easy to see that,

$$
\|x+y\|^{p}=\|x\|^{p}+\|y\|^{p}
$$

and

$$
\left\|V_{0}(x)+V_{0}(y)\right\|^{p}=\left\|V_{0}(x)\right\|^{p}+\left\|V_{0}(y)\right\|^{p}
$$

for every $x, y \in S(E)$. Where, $E$ is a subspace of the space $s_{p}(\alpha, H)$ in which for every $x$ and $y$ of $E$ if supp $x \cap \operatorname{supp} y=\emptyset$ then above relation (2.8) hold.

Lemma 2.6. Let $E$ be a subspace of the space $s_{p}(\alpha, H)$ in which for every $x$ and $y$ of $E$ if supp $x \cap$ supp $y=\emptyset$ then above relation (2.8) hold. Suppose that $V_{0}$ is an isometric mapping on the unit sphere $S(E)$ into itself. If $x$ and $y$ are two disjoint elements of $S(E), \xi$ and $\eta$ are real numbers and $z=\xi x+\eta y \in S(E)$, then

$$
V_{0}(z)=\xi V_{0}(x)+\eta V_{0}(y)
$$

Proof. By the hypothesis of the subspace $E$ of $s_{p}(\alpha, H)$, we have above relation (2.8). Hence, if we put

$$
A=\operatorname{supp} V_{0}(x), B=\operatorname{supp} V_{0}(y)
$$

Remark 2.5 implies that

$$
\left\|\left.V_{0}(z)\right|_{A}\right\|^{p}+\left\|\left.V_{0}(z)\right|_{B}\right\|^{p}=\left\|\left.V_{0}(z)\right|_{A \cup B}\right\|^{p} \leq\left\|\left.V_{0}(z)\right|_{\Omega}\right\|^{p}=1,
$$

where $\Omega=\bigcup_{x \in E} \operatorname{supp} x$.

If $\xi=0$ then $\eta=1$ or $\eta=-1$, so the relation $V_{0}(z)=\eta V_{0}(y)$ follows directly or by Lemma 2.1. The case when $\eta=0$ is dual.

Now assume that $\xi \neq 0$ and $\eta \neq 0$. From Lemma 2.1, Remark 2.5 and the hypothesis of $x$ and $y$, we obtain 


$$
\begin{aligned}
\left\|V_{0}(z)+\frac{\xi}{|\xi|} V_{0}(x)\right\|^{p} & =\left\|z+\frac{\xi}{|\xi|} x\right\|^{p} \\
& =\left\|\xi x+\frac{\xi}{|\xi|} x\right\|^{p}+\|\eta y\|^{p} \\
& =\left\|\frac{\xi}{|\xi|}(1+|\xi|) x\right\|^{p}+\|\eta y\|^{p} \\
& =(1+|\xi|)^{p}+1-|\xi|^{p},
\end{aligned}
$$

where in the last equality we have used the the following identity

$$
1^{p}=\|z\|^{p}=\|\xi x+\eta y\|^{p}=\|\xi x\|^{p}+\|\eta y\|^{p}=|\xi|^{p}+|\eta|^{p},
$$

which follows from the assumption supp $x \cap \operatorname{supp} y=\emptyset$ and Remark 2.5.

Similarly, we also have

$$
\left\|V_{0}(z)+\frac{\eta}{|\eta|} V_{0}(y)\right\|^{p}=(1+|\eta|)^{p}+1-|\eta|^{p} .
$$

On the other hand, we have

$$
\left\|V_{0}(z)+\frac{\xi}{|\xi|} V_{0}(x)\right\|^{p}=\left\|\left.\left(V_{0}(z)+\frac{\xi}{|\xi|} V_{0}(x)\right)\right|_{A}+\left.\left(V_{0}(z)+\frac{\xi}{|\xi|} V_{0}(x)\right)\right|_{\Omega-A}\right\|^{p} .
$$

Notice the hypothesis of the subspace $E$ of $s_{p}(\alpha, H)$ and the definition of the norm of $s_{p}(\alpha, H)$, along with Remark 2.5, imply

$$
\begin{aligned}
& \left\|\left.\left(V_{0}(z)+\frac{\xi}{|\xi|} V_{0}(x)\right)\right|_{A}+\left.\left(V_{0}(z)+\frac{\xi}{|\xi|} V_{0}(x)\right)\right|_{\Omega-A}\right\|^{p} \\
= & \left\|\left.\left(V_{0}(z)+\frac{\xi}{|\xi|} V_{0}(x)\right)\right|_{A}\right\|^{p}+\left\|\left.\left(V_{0}(z)+\frac{\xi}{|\xi|} V_{0}(x)\right)\right|_{\Omega-A}\right\|^{p} \\
\leq & \left(\left\|\left.V_{0}(z)\right|_{A}\right\|+\left\|\left.\frac{\xi}{|\xi|} V_{0}(x)\right|_{A}\right\|\right)^{p}+\left\|\left.V_{0}(z)\right|_{\Omega-A}\right\|^{p} \\
= & \left(1+\|\left. V_{0}(z)\right|_{A}||\right)^{p}+1-\left\|\left.V_{0}(z)\right|_{A}\right\|^{p} .
\end{aligned}
$$

That is,

$$
\left\|V_{0}(z)+\frac{\xi}{|\xi|} V_{0}(x)\right\|^{p} \leq\left(1+\|\left. V_{0}(z)\right|_{A}||\right)^{p}+1-\left\|\left.V_{0}(z)\right|_{A}\right\|^{p} .
$$

Similarly, we also obtain

$$
\left\|V_{0}(z)+\frac{\eta}{|\eta|} V_{0}(y)\right\|^{p} \leq\left(1+\left\|\left.V_{0}(z)\right|_{B}\right\|\right)^{p}+1-\left\|\left.V_{0}(z)\right|_{B}\right\|^{p} .
$$

Hence, by (2.10) and ((2.13),

$$
(1+|\xi|)^{p}+1-|\xi|^{p} \leq\left(1+\|\left. V_{0}(z)\right|_{A}||\right)^{p}+1-\left\|\left.V_{0}(z)\right|_{A}\right\|^{p}
$$

and also by (2.12) and (2.14) we have

$$
(1+|\eta|)^{p}+1-|\eta|^{p} \leq\left(1+\left.|| V_{0}(z)\right|_{B}||\right)^{p}+1-\left\|\left.V_{0}(z)\right|_{B}\right\|^{p} .
$$

Notice that the real function $\varphi(t)=(1+t)^{p}+1-t^{p}$ is strictly increasing in $[0, \infty)$, hence (2.15) and (2.16) imply

$$
|\xi| \leq\left\|\left.V_{0}(z)\right|_{A}\right\| \text { and }|\eta| \leq\left\|\left.V_{0}(z)\right|_{B}\right\| \text {. }
$$


By (2.9), (2.11) and (2.17), we obtain

$$
|\xi|=\left\|\left.V_{0}(z)\right|_{A}\right\| \text { and }|\eta|=\left\|\left.V_{0}(z)\right|_{B}\right\| .
$$

From (2.9),(2.11) and (2.18), we obtain

$$
\left\|\left.V_{0}(z)\right|_{\Omega}\right\|^{p}=\left\|\left.V_{0}(z)\right|_{A}\right\|^{p}+\left\|\left.V_{0}(z)\right|_{B}\right\|^{p}
$$

Therefore supp $\left.V_{0}(z)\right|_{\Omega-A \cup B}=\emptyset$, and so

$$
V_{0}(z)=\left.V_{0}(z)\right|_{A}+\left.V_{0}(z)\right|_{B}
$$

By (2.10), (2.11),(2.18) and (2.19), we have

$$
\begin{aligned}
& (1+|\xi|)^{p}+|\eta|^{p} \\
= & \left\|V_{0}(z)+\frac{\xi}{|\xi|} V_{0}(x)\right\|^{p} \\
\leq & \left\|\left.V_{0}(z)\right|_{A}+\frac{\xi}{|\xi|} V_{0}(x)\right\|^{p}+\left\|\left.V_{0}(z)\right|_{B}\right\|^{p} \\
\leq & \left(1+\left.|| V_{0}(z)\right|_{A}||\right)^{p}+\left\|\left.V_{0}(z)\right|_{B}\right\|^{p} \\
= & (1+|\xi|)^{p}+|\eta|^{p}
\end{aligned}
$$

which implies

$$
\left\|\left.V_{0}(z)\right|_{A}+\frac{\xi}{|\xi|} V_{0}(x)\right\|=\left\|\left.V_{0}(z)\right|_{A}\right\|+\left\|\frac{\xi}{|\xi|} V_{0}(x)\right\| .
$$

Since $s_{p}(\alpha, H)$ is strictly convex, we have $\left.V_{0}(z)\right|_{A}=c \frac{\xi}{|\xi|} V_{0}(x)$ for some $c>0$, and $c=|\xi|$ because of (2.18). That is, $\left.V_{0}(z)\right|_{A}=\xi V_{0}(x)$. Similarly, we also have $\left.V_{0}(z)\right|_{B}=\eta V_{0}(x)$. Thus we obtain $V_{0}(z)=\xi V_{0}(x)+\eta V_{0}(y)$. We have completed the proof.

Lemma 2.7. Let $E$ be a subspace of the space $s_{p}(\alpha, H)$ in which for every $x$ and $y$ of $E$ if supp $x \cap$ supp $y=\emptyset$ then relation (2.8) hold. Suppose that $V_{0}$ is an isometric mapping on the unit space $S(E)$ into itself. If $x_{1}, x_{2}, \cdots, x_{m}$ are mutual disjoint elements of $S(E)$ and $\sum_{n=1}^{m} \xi_{n} x_{n} \in S(E)$, then

$$
V_{0}\left(\sum_{n=1}^{m} \xi_{n} x_{n}\right)=\sum_{n=1}^{m} \xi_{n} V_{0}\left(x_{n}\right)
$$

Proof. We prove this by induction. Indeed, for $m=2$ the result holds by Lemma 2.6. If for $m=m_{0}-1$ the results holds. For $m=m_{0}$, by using the following equality

$$
\sum_{n=1}^{m_{0}} \xi_{n} x_{n}=\xi_{1} x_{1}+\left\|\sum_{n=2}^{m_{0}} \xi_{n} x_{n}\right\| \cdot \sum_{n=2}^{m_{0}} \frac{\xi_{n}}{\left\|\sum_{n=2}^{m_{0}} \xi_{n} x_{n}\right\|} x_{n}
$$

and the hypothesis of the subspace $E$, where at least one $\xi_{n}$ for $n \geq 2$ is a nonzero number, we get the desired result. If all $\xi_{n}$ are zero for $n \geq 2$, then $\left|\xi_{1}\right|=1$, so $V_{0}\left(\xi_{1} x_{1}\right)=\xi_{1} V_{0}\left(x_{1}\right)$ and we are done. 
Lemma 2.8. Suppose that $E$ is a subspace of the space $s_{p}(\alpha, H)$ satisfying (1.4) and (1.5) and that $V_{0}$ is an isometric mapping from the unit sphere $S(E)$ onto itself. Then, for any $e_{x(n)} \in S(E)$, supp $V_{0} e_{x(n)}$ is a single point set.

Proof. It is easy to see that the result of Lemma 2.3 and relation (2.8) hold for the space $E$. Without loss of generality, we can assume

$$
V_{0}\left(e_{x(1)}\right)=e_{x^{\prime}(k)}+\sum_{i \neq k} e_{x^{\prime}(i)}
$$

where $x^{\prime}(k) \neq o$. Let

$$
y=\frac{e_{x^{\prime}(k)}}{\left\|e_{x^{\prime}(k)}\right\|} \text {. }
$$

Since $V_{0}$ is surjective, there exists $u \in S(E)$ such that $V_{0} u=y$. By Lemma 2.3,

$$
\begin{aligned}
\left(\operatorname{supp} V_{0} u\right) \bigcap\left(\operatorname{supp} V_{0} e_{x(n)}\right) & =(\operatorname{suppy}) \bigcap\left(\operatorname{supp} V_{0} e_{x(n)}\right) \\
& \subseteq\left(\operatorname{supp} V_{0} e_{x(1)}\right) \bigcap\left(\operatorname{supp} V_{0} e_{x(n)}\right)=\emptyset
\end{aligned}
$$

holds for any $n \neq 1$, where $x(n) \neq 0$.

Applying Lemma 2.3 again,we have

$$
(\text { suppu }) \bigcap\left(\operatorname{suppe}_{x(n)}\right)=\emptyset(n \neq 1) .
$$

This means that $u=e_{x(1)}$, and this implies $\operatorname{Supp}_{0}\left(e_{x(1)}\right)=\{k\}$.

Lemma 2.9. Suppose that $x_{1}$ and $y_{1}$ are elements in the Hilbert space $H$, that $\lambda$ and $\mu$ are non - zero real numbers, that $\left\|\lambda x_{1} \pm \mu y_{1}\right\|=\left\|\lambda x_{2} \pm \mu y_{2}\right\|$, that $\left\|x_{1}\right\|=\left\|x_{2}\right\|$ and that $\left\|y_{1}\right\|=\left\|y_{2}\right\|$. Then $\left\|x_{1}-y_{1}\right\|=\left\|x_{2}-y_{2}\right\|$.

Proof. It is easy to prove that by the parallelogram law.

Now we are in a position to state the main results and proofs in this paper.

Theorem 2.10. Suppose that $E$ is a subspace of the space $s_{p}(\alpha, H)$ satisfying (1.4) and (1.5), that $1 \notin S u p p E$ and that $V_{0}$ is an isometric mapping from the unit sphere $S(E)$ onto itself. Then $V_{0}$ can be extended to an isometry on the whole space $E$.

Proof. By Lemma 2.7, we see that for every $x=\{x(n)\}_{n=1}^{n=m} \in S(E)$, we have

$$
V_{0}(x)=\sum_{n=1}^{m}\left\|e_{x(n)}\right\| V_{0}\left(\frac{e_{x(n)}}{\left\|e_{x(n)}\right\|}\right) .
$$

If $\|x(n)\|=0$, then $V_{0}\left(\frac{e_{x(n)}}{\left\|e_{x(n)}\right\|}\right) \stackrel{\text { def }}{=} 0$.

We now define a mapping on the subspace $E_{0}$ of $E$ which consists of all elements in which every element only has finitely many non-zero items as follows

$$
V_{1}\left(\{x(n)\}_{n=1}^{n=m}\right) \stackrel{\text { def }}{=} \sum_{n=1}^{m}\left\|e_{x(n)}\right\| V_{0}\left(\frac{e_{x(n)}}{\left\|e_{x(n)}\right\|}\right)
$$

for all $\{x(n)\}_{n=1}^{n=m} \in E_{0} \subset E$. 
Suppose that $\{x(n)\}_{n=1}^{n=m}$ and $\{y(n)\}_{n=1}^{n=m}$ are elements in $E_{0}$. By lemma 2.8, we can assume

and

$$
V_{1}\left(\{x(n)\}_{n=1}^{n=m}\right)=\left\{x^{\prime}(k(n))\right\}_{n=1}^{n=m}
$$

To prove

$$
V_{1}\left(\{y(n)\}_{n=1}^{n=m}\right)=\left\{y^{\prime}(k(n))\right\}_{n=1}^{n=m}
$$

$$
\left\|V_{1}\left(\{x(n)\}_{n=1}^{n=m}\right)-V_{1}\left(\{y(n)\}_{n=1}^{n=m}\right)\right\|=\left\|\{x(n)\}_{n=1}^{n=m}-\{y(n)\}_{n=1}^{n=m}\right\| .
$$

We proceed as follows

$$
\begin{aligned}
& \left\|V_{0}\left(\frac{e_{x(n)}}{\left\|e_{x(n)}\right\|}\right) \pm V_{0}\left(\frac{e_{y(n)}}{\left\|e_{y(n)}\right\|}\right)\right\|^{p} \\
= & \left\|\frac{e_{x(n)}}{\left\|e_{x(n)}\right\|} \pm \frac{e_{y(n)}}{\left\|e_{y(n)}\right\|}\right\|^{p} \\
= & \left(1+2^{p} \alpha\right)\left\|\frac{x(n)}{\left\|e_{x(n)}\right\|} \pm \frac{y(n)}{\left\|e_{y(n)}\right\|}\right\|^{p}
\end{aligned}
$$

On the other hand

$$
\begin{gathered}
\left\|V_{0}\left(\frac{e_{x(n)}}{\left\|e_{x(n)}\right\|}\right) \pm V_{0}\left(\frac{e_{y(n)}}{\left\|e_{y(n)}\right\|}\right)\right\|^{p} \\
=\left\|\frac{e_{x^{\prime}(k(n))}}{\left\|e_{x^{\prime}(k(n))}\right\|} \pm \frac{e_{y^{\prime}(k(n))}}{\left\|e_{y^{\prime}(k(n))}\right\|}\right\|^{p} \\
=\left(1+2^{p} \alpha\right)\left\|\frac{x^{\prime}(k(n))}{\left\|e_{x^{\prime}(k(n))}\right\|} \pm \frac{y^{\prime}(k(n))}{\left\|e_{y^{\prime}(k(n))}\right\|}\right\|^{p} .
\end{gathered}
$$

If $\|x(n)\|=0$, then $\frac{x(n)}{\left\|e_{x(n)}\right\|} \stackrel{\text { def }}{=} 0$ and $\frac{x^{\prime}(k(n))}{\left\|e_{x^{\prime}(k(n))}\right\|} \stackrel{\text { def }}{=} 0$. It follows from (2.23) and (2.24) that

$$
\left\|\frac{x(n)}{\left\|e_{x(n)}\right\|} \pm \frac{y(n)}{\left\|e_{y(n)}\right\|}\right\|=\left\|\frac{x^{\prime}(k(n))}{\left\|e_{x^{\prime}(k(n))}\right\|} \pm \frac{y^{\prime}(k(n))}{\left\|e_{y^{\prime}(k(n))}\right\|}\right\|
$$

Notice that $\left\|e_{x(n)}\right\|=\left\|e_{x^{\prime}(k(n))}\right\|$, that $\left\|e_{y(n)}\right\|=\left\|e_{y^{\prime}(k(n))}\right\|$ and (2.25), it follows that from Lemma 2.9 that

Since

$$
\|x(n)-y(n)\|=\| x^{\prime}(k(n))-y^{\prime}(k(n) \|
$$

$$
\left\|V_{1}\left(\{x(n)\}_{n=1}^{n=m}\right)-V_{1}\left(\{y(n)\}_{n=1}^{n=m}\right)\right\|^{p}=\sum_{n=1}^{m}\left(1+2^{p} \alpha\right)\left\|x^{\prime}(k(n))-y^{\prime}(k(n))\right\|^{p}(2
$$

and

$$
\left\|\{x(n)\}_{n=1}^{n=m}-\{y(n)\}_{n=1}^{n=m}\right\|^{p}=\sum_{n=1}^{m}\left(1+2^{p} \alpha\right)\|x(n)-y(n)\|^{p} .
$$

(2.26), (2.27) and (2.28) assure (2.22) holds. That is we have obtained an isometry on the subspace $E_{0}$ of $E$. It is evident that $E_{0}$ is dense in the space $E, E$ is a Banach space and $V_{1}$ is isometric on $E_{0}$. Hence $V_{1}$ has a unique linear isometric extension $V$ on the whole space $E$. Thus $V$ is the desired extension of $V_{0}$ and the proof is complete. 
Theorem 2.11. Let $V_{0}$ be a Lamperti isometric mapping on the unit sphere $S\left(s_{p}(\alpha, H)\right)$ into itself. Then $V_{0}$ can be extended to an isometry on the whole space $s_{p}(\alpha, H)$.

Proof. We notice that, if $V_{0}$ is a Lamperti isometric mapping on the subset of the space $s_{p}(\alpha, H)$, then $V_{0}$ is also a isometric mapping on the same subset of the space $l_{p}(H)$. By Lemmas 2.1 and 2.3, we can obtain the simple conclusion like Lemma 2.6 as follows (and much easy because, for every disjoint $x$ and $y$ in $l^{p}(H)$, we have that $\left.\|x+y\|^{p}=\|x\|^{p}+\|y\|^{p}\right)$.

Similarly, for every such element $\{x(n)\}_{n=1}^{n=m} \in S\left(s_{p}(\alpha, H)\right)$, we have

$$
V_{0}\left(\{x(n)\}_{n=1}^{n=m}\right)=\sum_{n=1}^{m}\left\|e_{x(n)}\right\| V_{0}\left(\frac{e_{x(n)}}{\left\|e_{x(n)}\right\|}\right) .
$$

If $\|x(n)\|=0$, then $V_{0}\left(\frac{e_{x(n)}}{\left\|e_{x(n)}\right\|}\right) \stackrel{\text { def }}{=} 0$. Now we define a mapping on the whole space $s_{p}(\alpha, H)$ as follows

$$
V x= \begin{cases}\|x\| V_{0}\left(\frac{x}{\|x\|}\right), & \text { if } x \neq 0 \\ 0, & \text { if } x=0\end{cases}
$$

Then it is evident that

$$
\|V(x)\|=\|x\|, \quad \forall x \in s_{p}(\alpha, H) .
$$

By (2.29) and (2.30) for every such element $x=\{x(n)\}_{n=1}^{n=m} \in s_{p}(\alpha, H)$, we have that

$$
\begin{aligned}
V\left(\{x(n)\}_{n=1}^{n=m}\right) & =\|x\| V_{0}\left(\frac{x}{\|x\|}\right) \\
& =\sum_{n=1}^{m}\left\|e_{x(n)}\right\| V_{0}\left(\frac{e_{x(n)}}{\left\|e_{x(n)}\right\|}\right) .
\end{aligned}
$$

That is, we obtain an isometry on the subspace $E$ of $s_{p}(\alpha, H)$ in which every element only has finite many non-zero items since (2.30) and (2.31) hold. Since $E$ is dense in $s_{p}(\alpha, H)$ and $V$ is continuous mapping on $s_{p}(\alpha, H)$, we see that $V$ is an isometry on $s_{p}(\alpha, H)$ and it is the extension of $V_{0}$.

Acknowledgement. The author is supported by the project of Department of Education of Guangdong Province (No.2012KJCX0096), NNSF of China(11001107).

\section{REFERENCES}

1. G. An, Isometries on unit sphere of $\left(\ell^{\beta_{n}}\right)$, J. Math. Anal. Appl. 301 (2005), 249-254.

2. S. Campbell, Nontrivial isometries on $s_{p}(\alpha)$, Inter. J. Math. Sci. 5 (1982), 257-261.

3. G. Ding, The 1-Lipschitz mapping between the unit spheres of two Hilbert spaces can be extend to a real linear isometry of the whole space, Sci. China 45 (2002), 479-483.

4. R. Fleming and J. Jamison, Isometries of $s_{p}(\alpha)$, Canad. J. Math. 33 (1981), 59-67.

5. X. Fu, Isometries on the space s, Acta Math. Sci. 26B (2006), 502-508.

6. X. Fu and S. Li, Some properties of $l^{p}(A, X)$ spaces, Abstr. Anal. Appl. 2009, Article ID 562507, (2009), 8 pages.

7. X. Fu, The isometric extension of the into mapping from the unit sphere $S_{1}(E)$ to $S_{1}\left(l^{\infty}(\Gamma)\right)$, Acta Math. Sin. (Engl. Ser.) 24 (2008), no. 9, 1475-1482. 
8. X. Fu and S. Stevic, The problem of isometric extension in the unit sphere of the space $s_{p}(\alpha)$, Nonlinear Anal. 74 (2011), 733-738.

9. P. Mankiewicz, On extension of isometries in normed linear spaces, Bull. Acad. Polon. Sci. 20 (1972), 367-371.

10. R. Megginson, An Introduction to Banach Space Theory, Springer-Verlag, New York, 2003.

11. S. Mazur and S. Ulam, Sur les transformations isometriques d'espaces vectoriels normés, C. R. Acad. Sci. Paris, 194 (1932), 946-948.

12. C. Park and Th.M. Rassias Isometric additive mappings in generalized quasi-Banach spaces, Banach J. Math. Anal. 2 (2008), no.1, 59-69.

13. H. Royden, Real Analysis, The MacMillan Co., New York, 1968.

14. T. Rassias, Properties of isometric mappings, J. Math. Anal. Appl. 235 (1999), 108-121.

15. E.A. Sanchez Perez and D. Werner The geometry of Lp-spaces over atomless measure spaces and the Daugavet property, Banach J. Math. Anal. 5 (2011), no.1, 167-180.

16. D. Tingley, Isometries of the unit spheres, Geometriae Dedicata. 22 (1987), 371-378.

17. L. Tan and S. Xiang, On the Aleksandrov-Rassias problem and the Hyers-Ulam-Rassias stability problem, Banach J. Math. Anal.1 (2007),no. 1, 11-22.

Department of Mathematics, University of Jiaying, Meizhou 514015, GuangDONG, CHINA.

E-mail address: gdfuxh@sina.com 